\title{
DATA DEPENDENCE FOR THE AMPLITUDE EQUATION OF SURFACE WAVES
}

\author{
PAOLO SECCHI
}

\begin{abstract}
We consider the amplitude equation for nonlinear surface wave solutions of hyperbolic conservation laws. This is an asymptotic nonlocal, Hamiltonian evolution equation with quadratic nonlinearity. For example, this equation describes the propagation of nonlinear Rayleigh waves [7], surface waves on current-vortex sheets in incompressible MHD [1,2] and on the incompressible plasma-vacuum interface [13].

The local-in-time existence of smooth solutions to the Cauchy problem for the amplitude equation in noncanonical variables was shown in $[9,13]$. In the present paper we prove the continuous dependence in strong norm of solutions on the initial data. This completes the proof of the well-posedness of the problem in the classical sense of Hadamard.
\end{abstract}

\section{INTRODUCTION}

In the present paper we consider the following equation

$$
\varphi_{t}+\frac{1}{2} \mathbb{H}\left[\Phi^{2}\right]_{x x}+\Phi \varphi_{x x}=0, \quad \Phi=\mathbb{H}[\varphi] .
$$

Here $\mathbb{H}$ denotes the Hilbert transform defined by

$$
\mathbb{H}[\varphi](x)=\frac{1}{\pi} \text { p.v. } \int_{-\infty}^{+\infty} \frac{\varphi(y)}{x-y} d y,
$$

and such that

$$
\mathcal{F}[\mathbb{H}[\varphi]]=-i \operatorname{sgn}(\mathrm{k}) \mathcal{F}[\varphi]
$$

for $\mathcal{F}$ denoting the Fourier transformation.

This is an asymptotic nonlocal, Hamiltonian evolution equation in one space dimension, with quadratic nonlinearity. For example, the equation describes the propagation of nonlinear Rayleigh waves [7], surface waves on current-vortex sheets in incompressible MHD [1,2] and on the incompressible plasma-vacuum interface [13], where in the vacuum part the electric and magnetic fields are ruled by the Maxwell equations. In a sense this is a canonical model equation for nonlinear surface wave solutions of hyperbolic conservation laws, analogous to the inviscid Burgers equation for bulk waves.

Equation (1) also admits the alternative spatial form

$$
\varphi_{t}+[\mathbb{H}, \Phi] \Phi_{x x}+\mathbb{H}\left[\Phi_{x}^{2}\right]=0,
$$

where $[\mathbb{H}, \Phi]$ is the commutator of $\mathbb{H}$ with multiplication by $\Phi$, see [10]. The alternative form (2) shows that (1) is an equation of first order, due to a cancelation of the second order spatial derivatives appearing in (1).

The local-in-time existence of smooth solutions to the Cauchy problem for the amplitude equation in noncanonical variables was shown in $[9,13]$. In the present paper we prove the continuous dependence in strong norm of solutions on the initial data. This completes the proof of the well-posedness of (1) in the classical sense of Hadamard, after existence and uniqueness. As written in Kato's paper [11], this part may be the most difficult one, when dealing with hyperbolic problems. Our method is somehow

Date: January 20, 2016.

2010 Mathematics Subject Classification. Primary: 76W05; Secondary: 35Q35, 76E17, 76E25, 35R35, 76B03.

Key words and phrases. Incompressible Magneto-Hydrodynamics, Maxwell equations, plasma-vacuum interface.

The author is supported by the national research project PRIN 2012 "Nonlinear Hyperbolic Partial Differential Equations, Dispersive and Transport Equations: theoretical and applicative aspects". 
inspired by Beirão da Veiga's perturbation theory for the compressible Euler equations [3, 4], and its application to the problem of convergence in strong norm of the incompressible limit, see [5, 12]. Instead of directly estimating the difference between the given solution and the solutions to the problems with approximating initial data, the main idea is to make a triangularization with the more regular solution to a suitably chosen close enough problem.

Numerical computations $[1,7]$ show that solutions of (1) form singularities in which the derivative $\varphi_{x}$ blows up, but $\varphi$ appears to remain continuous. As far as we know, the global existence of appropriate weak solutions is an open question.

1.1. The kernel. Equation (1) can be written as

$$
\hat{\varphi}_{t}(k, t)+i \operatorname{sgn}(\mathrm{k}) \int_{-\infty}^{+\infty} \Lambda(\mathrm{k}-\ell, \ell) \hat{\varphi}(\mathrm{k}-\ell, \mathrm{t}) \hat{\varphi}(\ell, \mathrm{t}) \mathrm{d} \ell=0, \quad \forall \mathrm{k} \neq 0,
$$

for $\hat{\varphi}=\mathcal{F}[\varphi]$, with the kernel $\Lambda$ defined by

$$
\Lambda(k, \ell)=-\frac{2|k+\ell||k||\ell|}{|k+\ell|+|k|+|\ell|} .
$$

$\Lambda(k, \ell)$ is perhaps the simplest kernel arising for surface waves. It satisfies the properties

$$
\begin{aligned}
& \Lambda(k, \ell)=\Lambda(\ell, k) \quad \text { (symmetry) } \\
& \Lambda(k, \ell)=\overline{\Lambda(-k,-\ell)} \quad \text { (reality), } \\
& \Lambda(\alpha k, \alpha \ell)=\alpha^{2} \Lambda(k, \ell) \quad \forall \alpha>0 \quad \text { (homogeneity), } \\
& \Lambda(k+\ell,-\ell)=\overline{\Lambda(k, \ell)} \quad \forall k, \ell \in \mathbb{R} \quad \text { (Hamiltonian). }
\end{aligned}
$$

The value 2 of the scaling exponent in $(5 \mathrm{c})$ is consistent with the dimensional analysis in [2] for surface waves. It is shown by Alì et al. [2] that (5d) is a sufficient condition for (3), in addition to (5a), (5b), to admit a Hamiltonian structure, see also [7, 8].

1.2. Noncanonical variables and existence of the solution. As in [9] we introduce the noncanonical dependent variable $\psi(x, t)$ defined by

$$
\psi(x, t)=\left|\partial_{x}\right|^{1 / 2} \varphi(x, t), \quad \hat{\psi}(k, t)=|k|^{1 / 2} \hat{\varphi}(k, t) .
$$

Then rewriting equation (3) in terms of $\psi$ gives

$$
\hat{\psi}_{t}(k, t)+i k \int_{-\infty}^{+\infty} S(k-\ell, \ell) \hat{\psi}(k-\ell, t) \hat{\psi}(\ell, t) d \ell=0, \quad \forall k \neq 0,
$$

with kernel $S$ given by

$$
S(k, \ell)=\frac{\Lambda(k, \ell)}{|k \ell(k+\ell)|^{1 / 2}}=-\frac{2|k \ell(k+\ell)|^{1 / 2}}{|k+\ell|+|k|+|\ell|}
$$

The definition of $S$ is extended by setting

$$
S(k, \ell)=0 \quad \text { if } k \ell=0 .
$$

$S$ obviously satisfies

$$
\begin{aligned}
& S(k, \ell)=S(\ell, k) \quad \text { (symmetry), } \\
& S(k, \ell)=\overline{S(-k,-\ell)} \quad \text { (reality), } \\
& S(\alpha k, \alpha \ell)=\alpha^{1 / 2} S(k, \ell) \quad \forall \alpha>0 \quad \text { (homogeneity), } \\
& S(k+\ell,-\ell)=\overline{S(k, \ell)} \quad \forall k, \ell \in \mathbb{R} \quad \text { (Hamiltonian). }
\end{aligned}
$$

Moreover $S$ satisfies

$$
|S(k-\ell, \ell)| \leq \min \left\{|k|^{1 / 2},|k-\ell|^{1 / 2},|\ell|^{1 / 2}\right\} .
$$

The corresponding spatial form of (6) is

$$
\partial_{t} \psi+\partial_{x} a(\psi, \psi)=0,
$$


where the bilinear form $a$ is defined by

$$
\widehat{a(\psi, \phi})(k, t)=\int_{-\infty}^{+\infty} S(k-\ell, \ell) \hat{\psi}(k-\ell, t) \hat{\phi}(\ell, t) d \ell .
$$

(10) has the form of a nonlocal Burgers equation, like (2.8) in [9], or (1.1) in [6].

We consider the initial value problem for the noncanonical equation (10), (11), supplemented by an initial condition

$$
\psi(x, 0)=\psi_{0}(x) .
$$

The existence and uniqueness of the solution to (10)-(12) easily follows by adapting the proof of Hunter [9] (given for the periodic setting) to our case.

Theorem 1. For any $\psi_{0} \in H^{s}(\mathbb{R}), s>2$, the initial value problem (10)-(12) has a unique local solution

$$
\psi \in C\left(I_{*} ; H^{s}(\mathbb{R})\right) \cap C^{1}\left(I_{*} ; H^{s-1}(\mathbb{R})\right)
$$

defined on the time interval $I_{*}=\left(-\tau_{*}, \tau_{*}\right)$, where

$$
\tau_{*}=\frac{1}{C_{s}\left\|\psi_{0}\right\|_{L^{2}(\mathbb{R})}^{1-2 / s}\left\|\psi_{0}\right\|_{H^{s}(\mathbb{R})}^{2 / s}},
$$

for a suitable constant $C_{s}$. Moreover $\psi$ satisfies

$$
\begin{gathered}
\|\psi(t)\|_{L^{2}}=\left\|\psi_{0}\right\|_{L^{2}}, \\
\|\psi(t)\|_{H^{s}(\mathbb{R})} \leq\left\|\psi_{0}\right\|_{H^{s}(\mathbb{R})}\left(1-C_{s}\left\|\psi_{0}\right\|_{L^{2}(\mathbb{R})}^{1-2 / s}\left\|\psi_{0}\right\|_{H^{s}(\mathbb{R})}^{2 / s}|t|\right)^{-s / 2}, \quad \forall t \in I_{*} .
\end{gathered}
$$

For the proof of Theorem 1 we refer the reader to $[9,13]$, see also [14].

1.3. The main result. Now we state the main result of this paper about the continuous dependence in strong norm of solutions on the initial data.

Theorem 2. Let $s>2$. Let us consider $\psi_{0} \in H^{s}(\mathbb{R})$ and a sequence $\left\{\psi_{0}^{n}\right\}_{n \in \mathbb{N}} \subset H^{s}(\mathbb{R})$ such that $\psi_{0}^{n} \rightarrow \psi_{0}$ strongly in $H^{s}(\mathbb{R})$, as $n \rightarrow+\infty$. Let $\psi \in C\left(I ; H^{s}(\mathbb{R})\right) \cap C^{1}\left(I ; H^{s-1}(\mathbb{R})\right)$ and $\psi^{n} \in C\left(I ; H^{s}(\mathbb{R})\right) \cap$ $C^{1}\left(I ; H^{s-1}(\mathbb{R})\right)$ be the solutions of $(10)$, (11), with initial data $\psi_{0}$ and $\psi_{0}^{n}$ respectively, provided by Theorem 1, where we may assume without loss of generality that all solutions are defined on a common time interval $I=[-T, T]$, with $0<T<\tau_{*}{ }^{1}$. Then

$$
\psi^{n} \rightarrow \psi \quad \text { strongly in } C\left(I ; H^{s}(\mathbb{R})\right) \text {, as } n \rightarrow+\infty .
$$

Notice that, using the a priori estimate (15) and standard arguments, it is rather easy to show the continuous dependence of solutions on the initial data in the topology of $C\left(I ; H^{s-\varepsilon}(\mathbb{R})\right)$, for all small enough $\varepsilon>0$. Instead, in Theorem 2 we prove the continuous dependence precisely in the topology of $C\left(I ; H^{s}(\mathbb{R})\right)$.

From Theorem 1 and Theorem 2 we obtain that the initial value problem (10)-(12) is well-posed in $H^{s}$ in the classical sense of Hadamard.

The rest of the paper is organized as follows. In Section 2 we introduce some notation and give preliminary technical results. In Section 3 we prove our main Theorem 2.

\footnotetext{
${ }^{1}$ This assumption is a consequence of the convergence $\left\|\psi_{0}^{n}\right\|_{H^{s}} \rightarrow\left\|\psi_{0}\right\|_{H^{s}}$ and the formula for the maximal time of existence (13).
} 


\section{Preliminary Results}

2.1. Notation. In the paper we denote by $C$ generic positive constants, that may vary from line to line or even inside the same formula.

For nonnegative real numbers $s$ we consider standard Sobolev spaces $H^{s}=H^{s}(\mathbb{R})$ normed by

$$
\|\psi\|_{H^{s}}=\left(\int_{-\infty}^{+\infty}\left(1+|k|^{2}\right)^{s}|\hat{\psi}(k)|^{2} d k\right)^{1 / 2}, \quad \hat{\psi}=\mathcal{F}[\psi] .
$$

We also introduce the homogeneous space $\dot{H}^{s}(\mathbb{R})$,

$$
\dot{H}^{s}=\dot{H}^{s}(\mathbb{R})=\left\{\psi: \mathbb{R} \rightarrow \mathbb{R}: \int_{-\infty}^{+\infty}|k|^{2 s}|\hat{\psi}(k)|^{2} d k<+\infty\right\} .
$$

As inner product and norm in $\dot{H}^{s}$, we use ${ }^{2}$

$$
\langle\psi, \phi\rangle_{s}=\int_{-\infty}^{+\infty}|k|^{2 s} \hat{\psi}(k) \hat{\phi}(-k) d k, \quad\|\psi\|_{s}=\left(\int_{-\infty}^{+\infty}|k|^{2 s}|\hat{\psi}(k)|^{2} d k\right)^{1 / 2} .
$$

In particular we have

$$
\|\psi\|_{L^{2}(\mathbb{R})}=\|\psi\|_{0}=\left(\int_{-\infty}^{+\infty}|\hat{\psi}(k)|^{2} d k\right)^{1 / 2} .
$$

2.2. The bilinear form. Let us consider the bilinear form $a$ defined by (11). We first recall the properties of $a$ proven in [9], here adapted to the case on the whole real line.

Proposition 3. Let $a(\psi, \phi)$ be defined by (11) and let $s>1$. Then

(a) $a: \dot{H}^{s} \times \dot{H}^{s} \rightarrow \dot{H}^{s}$ is a bounded symmetric bilinear form.

(b) For all $\psi, \phi \in \dot{H}^{s} \cap \dot{H}^{s+1}$,

$$
a(\psi, \phi)_{x}=a\left(\psi_{x}, \phi\right)+a\left(\psi, \phi_{x}\right)
$$

(c) For all $\psi, \phi, \Psi \in L^{2} \cap \dot{H}^{s}$,

$$
(\psi, a(\phi, \Psi))_{L^{2}}=(\phi, a(\Psi, \psi))_{L^{2}}
$$

and there is a constant $C>0$ such that

$$
\left|(\psi, a(\phi, \Psi))_{L^{2}}\right| \leq C\|\psi\|_{s}\|\phi\|_{0}\|\Psi\|_{0} .
$$

Proof. See [9].

For the sequel we fix a real number $0<\alpha<1$, needed for the estimate

$$
\left\||k|^{1 / 2} \hat{g}\right\|_{L^{1}} \leq C\|g\|_{H^{1+\alpha}} \quad \forall g \in H^{1+\alpha} .
$$

In the following proposition we give some more properties of $a$.

Proposition 4. (a) Given any $s \geq 0$ and a fixed real number $0<\alpha<1$, there exists a constant $C>0$ such that, for all $\psi, \phi \in \dot{H}^{s} \cap H^{1+\alpha}$,

$$
\|a(\psi, \phi)\|_{s} \leq C\left(\|\psi\|_{s}\|\phi\|_{H^{1+\alpha}}+\|\psi\|_{H^{1+\alpha}}\|\phi\|_{s}\right) .
$$

In particular, if $s>0$, there exists a constant $C>0$ such that, for all $\psi, \phi \in H^{s} \cap H^{1+\alpha}$,

$$
\|a(\psi, \phi)\|_{H^{s}} \leq C\left(\|\psi\|_{H^{s}}\|\phi\|_{H^{1+\alpha}}+\|\psi\|_{H^{1+\alpha}}\|\phi\|_{H^{s}}\right) .
$$

(b) There exists a constant $C>0$ such that, for all $\psi \in H^{2+\alpha}, \Psi \in H^{1}$,

$$
\left|\left(a(\psi, \Psi)_{x}, \Psi\right)_{L^{2}}\right| \leq C\|\psi\|_{2+\alpha}\|\Psi\|_{0}^{2} .
$$

\footnotetext{
${ }^{2}$ If $\phi$ is real then $\overline{\hat{\phi}(k)}=\hat{\phi}(-k)$.
} 
Proof. The proof of (a) is essentially given in [9] and we repeat it for the sake of reader's convenience. For the sake of brevity in the following calculations we don't write explicitly the dependence on time $t$. Using (11) we get

$$
\left.\|a(\psi, \phi)\|_{s}^{2}=\int_{-\infty}^{+\infty}|k|^{2 s} \mid \widehat{a(\psi, \phi}\right)\left.(k)\right|^{2} d k=\int_{-\infty}^{+\infty}|k|^{2 s}\left|\int_{-\infty}^{+\infty} S(k-\ell, \ell) \hat{\psi}(k-\ell) \hat{\phi}(\ell) d \ell\right|^{2} d k .
$$

For $s \geq 0$ and $k, \ell \in \mathbb{R}$, we have

$$
|k|^{s} \leq C_{s}\left(|k-\ell|^{s}+|\ell|^{s}\right) .
$$

Using this inequality and (9) in (24) we get

$$
\begin{aligned}
& \|a(\psi, \phi)\|_{s}^{2} \leq C \int_{-\infty}^{+\infty}\left|\int_{-\infty}^{+\infty}\left(|k-\ell|^{s}+|\ell|^{s}\right) \min \left\{|k|^{1 / 2},|k-\ell|^{1 / 2},|\ell|^{1 / 2}\right\}\right| \hat{\psi}(k-\ell) \hat{\phi}(\ell)|d \ell|^{2} d k \\
& \leq C \int_{-\infty}^{+\infty}\left|\int_{-\infty}^{+\infty}\left(|k-\ell|^{s}|\ell|^{1 / 2}+|k-\ell|^{1 / 2}|\ell|^{s}\right)\right| \hat{\psi}(k-\ell) \hat{\phi}(\ell)|d \ell|^{2} d k \\
& =C \int_{-\infty}^{+\infty}\left|\left(|k|^{s}|\hat{\psi}|\right) *\left(|k|^{1 / 2}|\hat{\phi}|\right)+\left(|k|^{1 / 2}|\hat{\psi}|\right) *\left(|k|^{s}|\hat{\phi}|\right)\right|^{2} d k,
\end{aligned}
$$

where we have introduced the convolution

$$
\hat{f} * \hat{g}(k)=\int_{-\infty}^{+\infty} \hat{f}(k-\ell) \hat{g}(\ell) d \ell .
$$

Using Young's inequality

$$
\|\hat{f} * \hat{g}\|_{L^{2}} \leq\|\hat{f}\|_{L^{2}}\|\hat{g}\|_{L^{1}}
$$

and (20), from (25) we readily obtain

$$
\begin{aligned}
& \|a(\psi, \phi)\|_{s} \leq C\left(\left\||k|^{s} \hat{\psi}\right\|_{L^{2}}\left\||k|^{1 / 2} \hat{\phi}\right\|_{L^{1}}+\left\||k|^{1 / 2} \hat{\psi}\right\|_{L^{1}}\left\||k|^{s} \hat{\phi}\right\|_{L^{2}}\right) \\
& \leq C\left(\|\psi\|_{s}\|\phi\|_{H^{1+\alpha}}+\|\psi\|_{H^{1+\alpha}}\|\phi\|_{s}\right)
\end{aligned}
$$

that is (21). The proof of (22) follows combining (21) for $s>0$ and for $s=0$.

In order to show (23) we proceed as follows. Using integrations by parts and the properties of $a$ given in Proposition 3 we compute that

$$
\begin{aligned}
& \left(a(\psi, \Psi)_{x}, \Psi\right)_{L^{2}}=-\left(a(\psi, \Psi), \Psi_{x}\right)_{L^{2}} \\
& =-\left(\psi, a\left(\Psi, \Psi_{x}\right)\right)_{L^{2}}=-\frac{1}{2}\left(\psi, a(\Psi, \Psi)_{x}\right)_{L^{2}}=\frac{1}{2}\left(\psi_{x}, a(\Psi, \Psi)\right)_{L^{2}} .
\end{aligned}
$$

Using (19) with $s=1+\alpha>1$ in (26) gives

$$
\left|\left(a(\psi, \Psi)_{x}, \Psi\right)_{L^{2}}\right| \leq C\left\|\psi_{x}\right\|_{1+\alpha}\|\Psi\|_{0}^{2}=C\|\psi\|_{2+\alpha}\|\Psi\|_{0}^{2},
$$

that is $(23)$.

We conclude this preliminary section with one commutator estimate that will be used in the proof of the main result Theorem 2 .

Proposition 5. Given any $s>2$ and a fixed real number $0<\alpha<1$, there exists a constant $C>0$ such that, for all $\psi \in H^{s} \cap H^{2+\alpha}, \Psi \in H^{s-1} \cap H^{1+\alpha}$,

$$
\left\|\left[\left\langle\partial_{x}\right\rangle^{s-1} ; a(\psi, \cdot)_{x}\right] \Psi\right\|_{L^{2}} \leq C\left(\|\psi\|_{H^{s}}\|\Psi\|_{H^{1+\alpha}}+\|\psi\|_{H^{2+\alpha}}\|\Psi\|_{H^{s-1}}\right),
$$

where $\left\langle\partial_{x}\right\rangle=\left(1+\left|\partial_{x}\right|^{2}\right)^{1 / 2}$ is the Fourier multiplier in the variable $x$, and the commutator denotes

$$
\left[\left\langle\partial_{x}\right\rangle^{s-1} ; a(\psi, \cdot)_{x}\right] \Psi=\left\langle\partial_{x}\right\rangle^{s-1}\left(a(\psi, \Psi)_{x}\right)-a\left(\psi,\left\langle\partial_{x}\right\rangle^{s-1} \Psi\right)_{x} .
$$


Proof. From the definition of the commutator we have that its Fourier transform is

$$
\begin{aligned}
& \left(\left[\left\langle\partial_{x}\right\rangle^{s-1} ; a(\psi, \cdot)_{x}\right] \Psi\right)^{\wedge}(k)=i k\langle k\rangle^{s-1} \widehat{a(\psi, \Psi)}(k)-i k\left(a\left(\psi,\left\langle\partial_{x}\right\rangle^{s-1} \Psi\right)\right)^{\wedge}(k) \\
& =i k \int_{-\infty}^{+\infty}\left(\langle k\rangle^{s-1}-\langle\ell\rangle^{s-1}\right) S(k-l, l) \hat{\psi}(k-\ell) \hat{\Psi}(\ell) d \ell .
\end{aligned}
$$

Using (9) and the estimates

$$
\begin{gathered}
|k| \leq|k-\ell|+|\ell|, \\
\left|\langle k\rangle^{s-1}-\langle\ell\rangle^{s-1}\right| \leq C|k-\ell|\left(\langle k\rangle^{s-2}+\langle\ell\rangle^{s-2}\right) \leq C|k-\ell|\left(\langle k-\ell\rangle^{s-2}+\langle\ell\rangle^{s-2}\right), \\
|k|\left|\langle k\rangle^{s-1}-\langle\ell\rangle^{s-1}\right| \leq C|k-\ell|\left(\langle k-\ell\rangle^{s-1}+\langle\ell\rangle^{s-1}\right),
\end{gathered}
$$

gives

$$
\begin{aligned}
& \left|\left(\left[\left\langle\partial_{x}\right\rangle^{s-1} ; a(\psi, \cdot)_{x}\right] \Psi\right)^{\wedge}(k)\right| \leq C \int_{-\infty}^{+\infty}|k-\ell|\left(\langle k-\ell\rangle^{s-1}+\langle\ell\rangle^{s-1}\right)|S(k-l, l)||\hat{\psi}(k-\ell) \hat{\Psi}(\ell)| d \ell \\
& \leq C \int_{-\infty}^{+\infty}|k-\ell|\left(\langle k-\ell\rangle^{s-1}+\langle\ell\rangle^{s-1}\right) \min \left\{|k|^{1 / 2},|k-\ell|^{1 / 2},|\ell|^{1 / 2}\right\}|\hat{\psi}(k-\ell) \hat{\Psi}(\ell)| d \ell \\
& \leq C \int_{-\infty}^{+\infty}\langle k-\ell\rangle^{s}|\hat{\psi}(k-\ell)||\ell|^{1 / 2}|\hat{\Psi}(\ell)|+|k-\ell|^{3 / 2}|\hat{\psi}(k-\ell)|\langle\ell\rangle^{s-1}|\hat{\Psi}(\ell)| d \ell \\
& =C\left(\left(\langle k\rangle^{s}|\hat{\psi}| *|k|^{1 / 2}|\hat{\Psi}|+|k|^{3 / 2}|\hat{\psi}| *\langle k\rangle^{s-1}|\hat{\Psi}|\right) .\right.
\end{aligned}
$$

Using again Young's inequality, (20) and Plancherel's theorem we get

$$
\begin{aligned}
& \left.\left\|\left[\left\langle\partial_{x}\right\rangle^{s-1} ; a(\psi, \cdot)_{x}\right] \Psi\right\|_{L^{2}} \leq C\left(\left\|\langle k\rangle^{s} \hat{\psi}\right\|_{L^{2}}\left\||k|^{1 / 2} \hat{\Psi}\right\|_{L^{1}}\right)+\left\||k|^{3 / 2} \hat{\psi}\right\|_{L^{1}}\left\|\langle k\rangle^{s-1} \hat{\Psi}\right\|_{L^{2}}\right) \\
& \leq C\left(\|\psi\|_{H^{s}}\|\Psi\|_{H^{1+\alpha}}+\|\psi\|_{H^{2+\alpha}}\|\Psi\|_{H^{s-1}}\right),
\end{aligned}
$$

that is $(27)$.

\section{Proof of Theorem 2}

From Theorem 1 we can easily obtain the following result.

Proposition 6. Under the assumptions of Theorem 1, given any $T$ such that $0<T<\tau_{*}$, there exist constants $K>0$ and $\beta>0$, depending on $T$ and $\left\|\psi_{0}\right\|_{H^{s}(\mathbb{R})}$, such that, for all initial data $\psi_{0}^{\prime} \in H^{s}(\mathbb{R})$ with $\left\|\psi_{0}^{\prime}-\psi_{0}\right\|_{H^{s}(\mathbb{R})} \leq \beta$, the solution $\psi^{\prime}$ of (10), (11), with initial data $\psi_{0}^{\prime}$, satisfies

$$
\left\|\psi^{\prime}(t)\right\|_{H^{s}(\mathbb{R})} \leq K\left\|\psi_{0}^{\prime}\right\|_{H^{s}(\mathbb{R})}, \quad \forall t \in[-T, T] .
$$

Proof. For any $T$ such that $0<T<\tau_{*}$, with $\tau_{*}$ given in (13), we define $\delta>0$ from

$$
1-C_{s}\left\|\psi_{0}\right\|_{L^{2}(\mathbb{R})}^{1-2 / s}\left\|\psi_{0}\right\|_{H^{s}(\mathbb{R})}^{2 / s} T=2 \delta .
$$

Let $\beta>0$ be such that, if $\left\|\psi_{0}^{\prime}-\psi_{0}\right\|_{H^{s}(\mathbb{R})} \leq \beta$ then

$$
1-C_{s}\left\|\psi_{0}^{\prime}\right\|_{L^{2}(\mathbb{R})}^{1-2 / s}\left\|\psi_{0}^{\prime}\right\|_{H^{s}(\mathbb{R})}^{2 / s} T \geq \delta .
$$

From (15) for $\psi^{\prime}$ we get (31) with $K=\delta^{-s / 2}$.

As a consequence of Proposition 6 and convergence of the sequence $\left\{\psi_{0}^{n}\right\}_{n \in \mathbb{N}}$ to $\psi_{0}$ in $H^{s}$, from now on we assume, without loss of generality, that the sequence of solutions $\left\{\psi^{n}\right\}_{n \in \mathbb{N}}$ is uniformly bounded in $H^{s}$ on the common time interval $I=[-T, T]$, i.e. there exists $K^{\prime}>0$ such that

$$
\left\|\psi^{n}(t)\right\|_{H^{s}(\mathbb{R})} \leq K\left\|\psi_{0}^{n}\right\|_{H^{s}(\mathbb{R})} \leq K^{\prime}, \quad \forall t \in I, \forall n .
$$

Proposition 7. Let $s>2$. Under the assumptions of Theorem 2 the sequence of solutions $\left\{\psi^{n}\right\}_{n \in \mathbb{N}}$ converges to $\psi$ strongly in $C\left(I ; H^{s-1}\right)$. 
Proof. We take the difference of equation (10) for $\psi$ and $\psi^{n}$ and use the properties of Proposition 3 to get the equation

$$
\left(\psi-\psi^{n}\right)_{t}+a\left(\psi+\psi^{n}, \psi-\psi^{n}\right)_{x}=0 .
$$

Using (23) we compute that

$$
\left|\frac{1}{2} \frac{d}{d t}\left\|\psi-\psi^{n}\right\|_{0}^{2}\right|=\left|\left(a\left(\psi+\psi^{n}, \psi-\psi^{n}\right)_{x}, \psi-\psi^{n}\right)_{L^{2}}\right| \leq C\left\|\psi+\psi^{n}\right\|_{H^{2+\alpha}}\left\|\psi-\psi^{n}\right\|_{0}^{2} .
$$

Let $\alpha>0$ be small enough so that $s \geq 2+\alpha$. It follows from (32) that the sequence $\left\{\psi^{n}\right\}_{n \in \mathbb{N}}$ is bounded in $H^{2+\alpha}$, uniformly for $t \in I$, and we get

$$
\left|\frac{1}{2} \frac{d}{d t}\left\|\psi-\psi^{n}\right\|_{0}^{2}\right| \leq C\left\|\psi-\psi^{n}\right\|_{0}^{2} .
$$

Applying Gronwall's lemma we obtain from (33)

$$
\left\|\left(\psi-\psi^{n}\right)(t)\right\|_{0}^{2} \leq e^{2 C T}\left\|\psi_{0}-\psi_{0}^{n}\right\|_{0}^{2} \quad t \in I,
$$

which gives the strong convergence in $C\left(I ; L^{2}\right)$. By interpolation and the uniform boundedness in $H^{s}$ we get

$$
\left\|\left(\psi-\psi^{n}\right)(t)\right\|_{H^{s-1}} \leq C\left\|\left(\psi-\psi^{n}\right)(t)\right\|_{H^{s}}^{1-1 / s}\left\|\left(\psi-\psi^{n}\right)(t)\right\|_{L^{2}}^{1 / s} \leq C\left\|\left(\psi-\psi^{n}\right)(t)\right\|_{L^{2}}^{1 / s}, \quad t \in I,
$$

which gives the thesis.

Remark 8. Obviously, by the similar argument with a finer interpolation we could prove the strong convergence of $\psi^{n}$ to $\psi$ in $C\left(I ; H^{s-\alpha}\right)$, for all small enough $\alpha>0$. However, this is useless for the following argument.

We take two spatial derivatives of (10) and obtain

$$
\psi_{x x t}+2 a\left(\psi, \psi_{x x}\right)_{x}=-2 a\left(\psi_{x}, \psi_{x}\right)_{x} .
$$

For convenience let us denote

$$
F=-2 a\left(\psi_{x}, \psi_{x}\right)_{x}
$$

Using Proposition 4 we compute

$$
\|F\|_{H^{s-2}} \leq 2\left\|a\left(\psi_{x}, \psi_{x}\right)\right\|_{H^{s-1}} \leq C\left\|\psi_{x}\right\|_{H^{1+\alpha}}\left\|\psi_{x}\right\|_{H^{s-1}} \leq C\|\psi\|_{H^{2+\alpha}}\|\psi\|_{H^{s}},
$$

for $\alpha>0$ such that $2+\alpha \leq s$, and we deduce that $F \in C\left(I ; H^{s-2}\right)$. Moreover $\psi_{0 x x}=\partial_{x}^{2} \psi_{0} \in H^{s-2}$. Given $\varepsilon>0$, let us take $\Psi_{0}^{\varepsilon} \in H^{s-1}, F^{\varepsilon} \in L^{1}\left(I ; H^{s-1}\right)$ such that

$$
\left\|\Psi_{0}^{\varepsilon}-\psi_{0 x x}\right\|_{H^{s-2}}+\left\|F^{\varepsilon}-F\right\|_{L^{1}\left(I ; H^{s-2}\right)} \leq \varepsilon .
$$

Let us consider the Cauchy problem

$$
\begin{cases}\Psi_{t}^{\varepsilon}+2 a\left(\psi, \Psi^{\varepsilon}\right)_{x}=F^{\varepsilon}, & x \in \mathbb{R}, t \in I, \\ \Psi^{\varepsilon}(x, 0)=\Psi_{0}^{\varepsilon}(x), & x \in \mathbb{R} .\end{cases}
$$

Lemma 9. Let $s>2$. For any $\varepsilon>0$, the Cauchy problem (37) has a unique solution $\Psi^{\varepsilon} \in C\left(I ; H^{s-1}\right)$ and

$$
\left\|\Psi^{\varepsilon}\right\|_{C\left(I ; H^{s-1}\right)} \leq e^{C\|\psi\|_{C\left(I ; H^{s}\right)} T}\left\{\left\|\Psi_{0}^{\varepsilon}\right\|_{H^{s-1}}+\left\|F^{\varepsilon}\right\|_{L^{1}\left(I ; H^{s-1}\right)}\right\} .
$$

Proof. We apply $\left\langle\partial_{x}\right\rangle^{s-1}$ to (37) and obtain

$$
\left(\left\langle\partial_{x}\right\rangle^{s-1} \Psi^{\varepsilon}\right)_{t}+2 a\left(\psi,\left\langle\partial_{x}\right\rangle^{s-1} \Psi^{\varepsilon}\right)_{x}+2\left[\left\langle\partial_{x}\right\rangle^{s-1} ; a(\psi, \cdot)_{x}\right] \Psi=\left\langle\partial_{x}\right\rangle^{s-1} F^{\varepsilon} .
$$

Applying Proposition 4 gives

$$
\left|\left(a\left(\psi,\left\langle\partial_{x}\right\rangle^{s-1} \Psi^{\varepsilon}\right)_{x},\left\langle\partial_{x}\right\rangle^{s-1} \Psi^{\varepsilon}\right)_{L^{2}}\right| \leq C\|\psi\|_{H^{2+\alpha}}\left\|\left\langle\partial_{x}\right\rangle^{s-1} \Psi^{\varepsilon}\right\|_{0}^{2}=C\|\psi\|_{H^{2+\alpha}}\left\|\Psi^{\varepsilon}\right\|_{H^{s-1}}^{2},
$$

and applying Proposition 5 gives

$$
\left\|\left[\left\langle\partial_{x}\right\rangle^{s-1} ; a(\psi, \cdot)_{x}\right] \Psi^{\varepsilon}\right\|_{L^{2}} \leq C\left(\|\psi\|_{H^{s}}\left\|\Psi^{\varepsilon}\right\|_{H^{1+\alpha}}+\|\psi\|_{H^{2+\alpha}}\left\|\Psi^{\varepsilon}\right\|_{H^{s-1}}\right) .
$$


Let $\alpha$ be small enough so that $s \geq 2+\alpha$. From (39)-(41) we get

$$
\left|\frac{1}{2} \frac{d}{d t}\left\|\Psi^{\varepsilon}\right\|_{H^{s-1}}^{2}\right| \leq C\|\psi\|_{H^{s}}\left\|\Psi^{\varepsilon}\right\|_{H^{s-1}}^{2}+\left\|F^{\varepsilon}\right\|_{H^{s-1}}\left\|\Psi^{\varepsilon}\right\|_{H^{s-1}}
$$

and applying the Gronwall's lemma we obtain (38). Given the a priori estimate (38), the existence and uniqueness of the solution follows by standard arguments.

Now we estimate the difference $\Psi^{\varepsilon}-\psi_{x x}$, which solves the linear problem

$$
\begin{cases}\left(\Psi^{\varepsilon}-\psi_{x x}\right)_{t}+2 a\left(\psi, \Psi^{\varepsilon}-\psi_{x x}\right)_{x}=F^{\varepsilon}-F, & x \in \mathbb{R}, t \in I, \\ \left(\Psi^{\varepsilon}-\psi_{x x}\right)(x, 0)=\Psi_{0}^{\varepsilon}(x)-\psi_{0 x x}(x), & x \in \mathbb{R} .\end{cases}
$$

Lemma 10. Let $s>2$. For any $\varepsilon>0$, the difference $\Psi^{\varepsilon}-\psi_{x x}$ satisfies the estimate

$$
\left\|\Psi^{\varepsilon}-\psi_{x x}\right\|_{C\left(I ; H^{s-2}\right)} \leq C \varepsilon .
$$

Proof. As for (38) we obtain

$$
\left\|\Psi^{\varepsilon}-\psi_{x x}\right\|_{C\left(I ; H^{s-2}\right)} \leq e^{C\|\psi\|_{C\left(I ; H^{s}\right)} T}\left\{\left\|\Psi_{0}^{\varepsilon}-\psi_{0 x x}\right\|_{H^{s-2}}+\left\|F^{\varepsilon}-F\right\|_{L^{1}\left(I ; H^{s-2}\right)}\right\} .
$$

Thus (43) follows from (36). is

Finally we estimate the difference between $\psi_{x x}^{n}$ and $\Psi^{\varepsilon}$. The difference of the corresponding problems

$$
\begin{cases}\left(\Psi^{\varepsilon}-\psi_{x x}^{n}\right)_{t}+2 a\left(\psi^{n}, \Psi^{\varepsilon}-\psi_{x x}^{n}\right)_{x}=G_{n, \varepsilon}, & x \in \mathbb{R}, t \in I, \\ \left(\Psi^{\varepsilon}-\psi_{x x}^{n}\right)(x, 0)=\Psi_{0}^{\varepsilon}(x)-\psi_{0 x x}^{n}(x), & x \in \mathbb{R},\end{cases}
$$

where we have set

$$
G_{n, \varepsilon}=2 a\left(\psi^{n}-\psi, \Psi^{\varepsilon}\right)_{x}+F^{\varepsilon}-F^{n}, \quad F^{n}=-2 a\left(\psi_{x}^{n}, \psi_{x}^{n}\right)_{x} .
$$

Lemma 11. Let $s>2$. For any $\varepsilon>0$, there exists $M(\varepsilon)>0$ such that, for any $n$ the difference $\Psi^{\varepsilon}-\psi_{x x}^{n}$ satisfies the estimate

$$
\begin{aligned}
& \left\|\left(\Psi^{\varepsilon}-\psi_{x x}^{n}\right)(t)\right\|_{H^{s-2}} \\
& \quad \leq C\left\{\varepsilon+\left\|\psi_{0}-\psi_{0}^{n}\right\|_{H^{s}}+M(\varepsilon)\left\|\psi-\psi^{n}\right\|_{C\left(I ; H^{s-1}\right)}+\left|\int_{0}^{t}\left\|\left(\psi-\psi^{n}\right)(\tau)\right\|_{H^{s}} d \tau\right|\right\},
\end{aligned}
$$

for any $t \in I$.

Proof. As for (44) we first obtain for every $t$

$$
\left\|\left(\Psi^{\varepsilon}-\psi_{x x}^{n}\right)(t)\right\|_{H^{s-2}} \leq e^{C\left\|\psi^{n}\right\|_{C\left(I ; H^{s}\right)} T}\left\{\left\|\Psi_{0}^{\varepsilon}-\psi_{0 x x}^{n}\right\|_{H^{s-2}}+\left|\int_{0}^{t}\left\|G_{n, \varepsilon}(\tau)\right\|_{H^{s-2}} d \tau\right|\right\} .
$$

Applying (21) we get for every $\tau$

$$
\begin{aligned}
& \left\|G_{n, \varepsilon}\right\|_{H^{s-2}} \leq 2\left\|a\left(\psi^{n}-\psi, \Psi^{\varepsilon}\right)_{x}\right\|_{H^{s-2}}+\left\|F^{\varepsilon}-F^{n}\right\|_{H^{s-2}} \\
& \leq C\left\|\psi^{n}-\psi\right\|_{H^{s-1}}\left\|\Psi^{\varepsilon}\right\|_{H^{s-1}}+\left\|F^{\varepsilon}-F\right\|_{H^{s-2}}+\left\|F-F^{n}\right\|_{H^{s-2}},
\end{aligned}
$$

and integrating this inequality in $\tau$ between 0 and $t$ gives

$$
\begin{aligned}
\left|\int_{0}^{t}\left\|G_{n, \varepsilon}\right\|_{H^{s-2}} d \tau\right| \leq C T M(\varepsilon)\left\|\psi^{n}-\psi\right\|_{C\left(I ; H^{s-1}\right)} & \\
& +\left|\int_{0}^{t}\left\|F^{\varepsilon}-F\right\|_{H^{s-2}} d \tau\right|+\left|\int_{0}^{t}\left\|F-F^{n}\right\|_{H^{s-2}} d \tau\right|,
\end{aligned}
$$

where we have denoted

$$
M(\varepsilon):=e^{C\|\psi\|_{C\left(I ; H^{s}\right)} T}\left\{\left\|\Psi_{0}^{\varepsilon}\right\|_{H^{s-1}}+\left\|F^{\varepsilon}\right\|_{L^{1}\left(0, T ; H^{s-1}\right)}\right\},
$$

that is the right-hand side of (38). On the other hand, we have

$$
\left\|F-F^{n}\right\|_{H^{s-2}} \leq 2\left\|a\left(\psi_{x}-\psi_{x}^{n}, \psi_{x}+\psi_{x}^{n}\right)\right\|_{H^{s-1}} \leq C\left\|\psi_{x}-\psi_{x}^{n}\right\|_{H^{s-1}}\left\|\psi_{x}+\psi_{x}^{n}\right\|_{H^{s-1}}
$$


for all $\tau$, which yields, thanks to the uniform boundedness of $\psi^{n}$ in $C\left(I ; H^{s}\right)$,

$$
\left|\int_{0}^{t}\left\|F-F^{n}\right\|_{H^{s-2}} d \tau\right| \leq C\left|\int_{0}^{t}\left\|\psi-\psi^{n}\right\|_{H^{s}} d \tau\right| .
$$

Using (36), (48), (49) we get

$$
\begin{aligned}
& \left\|\Psi_{0}^{\varepsilon}-\psi_{0 x x}^{n}\right\|_{H^{s-2}}+\left|\int_{0}^{t}\left\|G_{n, \varepsilon}(\tau)\right\|_{H^{s-2}} d \tau\right| \\
& \leq \varepsilon+\left\|\psi_{0}-\psi_{0}^{n}\right\|_{H^{s}}+C T M(\varepsilon)\left\|\psi^{n}-\psi\right\|_{C\left(I ; H^{s-1}\right)}+C\left|\int_{0}^{t}\left\|\left(\psi-\psi^{n}\right)(\tau)\right\|_{H^{s}} d \tau\right| .
\end{aligned}
$$

Using again the uniform boundedness of $\psi^{n}$ in $C\left(I ; H^{s}\right)$, from (47), (50) we get (46).

Proof of Theorem 2. Adding (43), (46) gives

$$
\begin{aligned}
& \left\|\left(\psi_{x x}-\psi_{x x}^{n}\right)(t)\right\|_{H^{s-2}} \leq\left\|\left(\Psi^{\varepsilon}-\psi_{x x}\right)(t)\right\|_{H^{s-2}}+\left\|\left(\Psi^{\varepsilon}-\psi_{x x}^{n}\right)(t)\right\|_{H^{s-2}} \\
& \leq C\left\{\varepsilon+\left\|\psi_{0}-\psi_{0}^{n}\right\|_{H^{s}}+M(\varepsilon)\left\|\psi-\psi^{n}\right\|_{C\left(I ; H^{s-1}\right.}+\left|\int_{0}^{t}\left\|\left(\psi-\psi^{n}\right)(\tau)\right\|_{H^{s}} d \tau\right|\right\} .
\end{aligned}
$$

Using the estimate

$$
\left\|\psi-\psi^{n}\right\|_{H^{s}} \leq\left\|\psi-\psi^{n}\right\|_{H^{s-1}}+\left\|\psi_{x x}-\psi_{x x}^{n}\right\|_{H^{s-2}},
$$

and applying the Gronwall lemma in (51) we obtain

$$
\left\|\psi_{x x}-\psi_{x x}^{n}\right\|_{C\left(I ; H^{s-2}\right)} \leq C\left\{\varepsilon+\left\|\psi_{0}-\psi_{0}^{n}\right\|_{H^{s}}+(M(\varepsilon)+1)\left\|\psi-\psi^{n}\right\|_{C\left(I ; H^{s-1}\right)}\right\} .
$$

Given any $\delta>0$, let $\varepsilon=\varepsilon(\delta)$ be such that $C \varepsilon<\delta / 3$. With this fixed $\varepsilon$ in $M(\varepsilon)$, and taking account of Proposition 7 , let $n_{0}$ be such that, for any $n \geq n_{0}$,

$$
C\left\{\left\|\psi_{0}-\psi_{0}^{n}\right\|_{H^{s}}+(M(\varepsilon)+1)\left\|\psi-\psi^{n}\right\|_{C\left(I ; H^{s-1}\right)}\right\}<\delta / 3 .
$$

It follows that

$$
\left\|\psi_{x x}-\psi_{x x}^{n}\right\|_{C\left(I ; H^{s-2}\right)}<2 \delta / 3 \quad \forall n \geq n_{0} .
$$

Combining again with Proposition 7 we get, for a suitable $n_{0}^{\prime} \geq n_{0}$,

$$
\left\|\psi-\psi^{n}\right\|_{C\left(I ; H^{s}\right)}<\delta \quad \forall n \geq n_{0}^{\prime},
$$

which concludes the proof of Theorem 2.

\section{REFERENCES}

[1] G. Alì and J. K. Hunter. Nonlinear surface waves on a tangential discontinuity in magnetohydrodynamics. Quart. Appl. Math., 61(3):451-474, 2003.

[2] G. Alì, J. K. Hunter, and D. F. Parker. Hamiltonian equations for scale-invariant waves. Stud. Appl. Math., 108(3):305$321,2002$.

[3] H. Beirão da Veiga. Data dependence in the mathematical theory of compressible inviscid fluids. Arch. Rational Mech. Anal., 119(2):109-127, 1992.

[4] H. Beirão da Veiga. Perturbation theorems for linear hyperbolic mixed problems and applications to the compressible Euler equations. Comm. Pure Appl. Math., 46(2):221-259, 1993.

[5] H. Beirão da Veiga. Singular limits in compressible fluid dynamics. Arch. Rational Mech. Anal., 128(4):313-327, 1994.

[6] S. Benzoni-Gavage. Local well-posedness of nonlocal Burgers equations. Differential Integral Equations, 22(3-4):303$320,2009$.

[7] M.F. Hamilton, Yu. A. Il'insky, and E. A. Zabolotskaya. Evolution equations for nonlinear Rayleigh waves. J. Acoust. Soc. Am., 97:891-897, 1995.

[8] J. K. Hunter. Nonlinear surface waves. In Current progress in hyberbolic systems: Riemann problems and computations (Brunswick, ME, 1988), pages 185-202. Amer. Math. Soc., 1989.

[9] J. K. Hunter. Short-time existence for scale-invariant Hamiltonian waves. J. Hyperbolic Differ. Equ., 3(2):247-267, 2006.

[10] J. K. Hunter. Nonlinear hyperbolic surface waves. In Nonlinear conservation laws and applications, volume 153 of IMA Vol. Math. Appl., pages 303-314. Springer, New York, 2011.

[11] T. Kato. Quasi-linear equations of evolution, with applications to partial differential equations. pages 25-70. Lecture Notes in Math., Vol. 448. Springer, Berlin, 1975. 
[12] P. Secchi. On the singular incompressible limit of inviscid compressible fluids. J. Math. Fluid Mech., 2(2):107-125, 2000.

[13] P. Secchi. Nonlinear surface waves on the plasma-vacuum interface. Quart. Appl. Math., 73(4):711-737, 2015.

[14] P. Secchi. On the amplitude equation of approximate surface waves on the plasma-vacuum interface. Proc. of the Int. Conference on Fluid Dynamics Past, Present and Future, 2015, to appear.

DiCATam, Mathematical Division, University of Brescia, Via Branze 43, 25123 Brescia, Italy

E-mail address: paolo.secchi@unibs.it 